\title{
Precision Medicine: Measurements without concepts are blind; concepts without observations are empty.
}

\author{
DONALD stanley ${ }^{1}$ \\ ${ }^{1}$ Maine Medical Center - Scarborough Campus
}

April 28, 2020

\begin{abstract}
What is precision medicine? No standard definition is found. Sometimes described as High Definition Medicine which is: "the dynamic assessment , management, and understanding of an individual's health measured at (or near) its most basic units." The impetus for this venture was advanced and stimulated by biogenetics, the study of how genes and their products affect health but also contribute to disease or resistance to cure. Medicine is an epistemology: a way of knowing, perceiving, remembering, finding out, proving, inferring, wondering, reflecting, a conceptual knowing relying upon observations fitted to disease concepts.
\end{abstract}

\section{Definition of Precision Medicine}

What is precision medicine? No standard definition is found. Sometimes described as High Definition Medicine which is: "the dynamic assessment, management, and understanding of an individual's healthmeasured at (or near) its most basic units." 11 The impetus for this venture was advanced and stimulated by biogenetics, the study of how genes and their products affect health but also contribute to disease or resistance to cure. Medicine is an epistemology: a way of knowing, perceiving, remembering, finding out, proving, inferring, wondering, reflecting, a conceptual knowing relying upon observationsfitted to disease concepts.

While precision medicine relies upon a mechanistic classification of disease based on biogenetics. Medical reductionism captures the sense in which precise measurements are construed as the conceptual basis of medical practice. This in turn relies upon artificial intelligence and so-called machine learning.22

We stipulate what a disease consists of by our sensory observations and intuit a concept. Immanuel Kant in the Critique of Pure Reason argued that

a priori synthetic judgments were necessary in order to understand all sensation. This type of judgment is independent of all other faculties.

Viral infections fall into a concept, as does autoimmune disease. The only advantage precision medicine offers is to turn these sensory level observations (e.g. an RNA polymerase that does not function correctly thereby preventing DNA messages to be transcribed) over to artificial intelligence and machine learning to describe, or discriminate, a pattern, a concept, in short, to create a new classification of disease, so-called online learning similar to what sellers on the internet do to make offers relevant to consumers.

But DNA is a completely passive biologic molecule and further measurement would only modify our concept of disease; we would require a polymerase to activate DNA. DNA acts only when it is signaled by theentire organism to produce RNA's which in turn form templates for the production of proteins. And less than $5 \%$ of DNA is able to participate in coding for protein production. Even if I knew what introns did, I could not use this information. Only coding and transmission via mRNA promotes life. DNA cannot be 
transmitted faithfully unless corrected by the entire organism. So there is nothing new under the genome! The ramifications would rely on 'deep' and exhaustive searching of the genome of which I know something, but not all functionality.

Keith Yamamoto coined the term precision medicine in 2010 as a broad concept in order to integrate rational, Data-driven mechanism-based health-care. So this new concept of molecular medicine would utilize the evolving collections of big-data, by employing artificial intelligence and machine-learning. Similar to facerecognition programs? Big data would be treated as possibly linking all similar data to machine learning then to disease classification, diagnosis and treatment? Is this feasible? No, because diseases evolve: what today represents population-based data will change as diseases change. See discussion below.

Moreover precision medicine relies upon big data and a form of 'digital health' as described by complex systems captured in machine learning. The definition of 'precision' is a deterministic approach to knowledge; not probabilistic, as in traditional medicine. Precision medicine is the aggregated collection, interpretation, and application of data to define all disease. But precision medicine is only a recent ambiguous name for improved diagnosis and treatment e.g. imaging developments CT with contrast, MRI, allow for more accurate staging of tumors; this might be thought of as precision medicine. By these methods we observe entities that heretofore were not seen. We observe what Galileo's telescope offered to churchmen.

Diseases evolve so population data changes:

Only biologic entities evolve. Traditionally we define a disease as a natural kind based on the probability of correct diagnosis (category) and cure when properly classified, staged and treated; a disease evolves even during treatment. Disease is a living entity that sometimes resists intervention: the purpose of clinical trials is to allow inductive inferences in order to classify and identify the disease that resists treatment and continues to live. (precision medicine may fill a role here only if it provides insight into the traditional categories of disease.). If disease were not a natural kind i.e. aberrations within a biological system, mimics of closely related diseases e.g. type 2 diabetes mellitus vs. insulin dependent diabetes, then these would be entities sui generis, a part of the world of organisms (a category, vegetable, animal). Our disease classification permits us to make inductions about what disease and what treatment. Not all diseases fit easily under one classification e.g. are prion diseases classified as infectious. The only possible contribution that precision medicine may contribute is increased specificity.

What is precision treatment and how did it change? Treatment follows an evolutionary course.

Syphilis was treated by mercury, then Salvarsan then Penicillin G.

Dropsy by leeches, then Digitalis, now treatment is aimed at underlying causes: IHD, Valvular disease, cardiomyopathy, hypertension, diabetes mellitus.

Diabetes mellitus by a low-starch diet and vigorous exercise, or an herbaceous blend of lupine (a legume with edible peas and gaudy flower spikes), fenugreek (a small herb with pungent yellow seeds) and zedoaria (a wetland crop whose roots taste like ginger with a bitter aftertaste, then insulin. Progress to GLP-1 for type 2 diabetes

All these are increases in diagnosis and treatment: This is precision medicine during the last 2.5 millennia. Thus the approach is same wine, new bottle.

Today the leading contenders for inclusion in precision medicine are suggested by biogenetics, microbiomics, epitope alterations, lifestyle, co-existing disease, ethnicity, sex, age, the factors that most physicians practicing medicine would consider in order to diagnose and treat disease. It is a holoscopic rather than meroscopic approach, as in precision medicine the use of artificial intelligence, relying on AI and a mechanistic appropriation of data points. But diseases evolve so population-based data require real-time processing as evolutionary changes occur.

Is precision medicine's mechanistic approach sufficiently different to change medicine? Has not the increase of specific treatment always been the aim? Is this not historically the case? 
Consider complex systems; 'the butterfly effect' Would the microbiome or the co-existence of depression, hypertension or diabetes influence this complex system? Would precision medicine make the categories more precise in e.g. DSM-5? ICD-10? Perhaps, and that is welcome as specificity has always been the aim. But AI and machine learning must account for evolutionary changes in disease. The data base would need continuous updating.

Precision medicine promises to establish more precise classifications for diseases, especially, recently, for malignant tumors, by applying more specific data derived from biogenetics. For example in a recent partnership between a pharmaceutical company and a cancer center, 10,000 cancer patients' tumors were further analyzed for actionable mutations that would suggest further clinical trials to identify treatment options. When subtypes of tumors were further classified there were too few identified similar tumor subtypes to proceed to a trial. Precision, perhaps? But too many classes. It might be possible for a cooperative study and new classification if recruitment of sufficient number of patients with a sub-subtype were to be identified nationwide or worldwide. Genetic tumor markers, permutations, identify classes of tumors that are not usually recognized in the ICD-10 classification and this may be the role of precision medicine? This in order to treat non-responders (the participants whose tumor markers (are different from the majority) in Phase III clinical trials.

On the contrary medicine promulgates that we ought to treat theperson with the disease and not the data points of a disease, not a mechanical application of data. Observations confined to data-point-generated concepts are blind. There is no disease without a concept, in spite of an array of data-points. Personalized medicine relies on a conceptual model of disease based on physical examination, pathophysiology, imaging, laboratory tests experience, and large clinical trials or meta-analyses of trials.

Without the conventional classification of diseases, e.g. ICD-10, precision medicine would entail innumerable classes of diseases. There would not be a class that contains 1.infectious 2.parasitic, 3.malignant, or 4.degenerative diseases. Each disease subtype would be in a separate class. There might be no class that contains all malignant lymphoma subtypes. Lymphoma now lists more than 60 subclasses.

Precision medicine would consider each patient's measurements to exhibit a separate distinct disease, a class made up of one patient by collecting innumerable distinctive data points of its own without overlap: no projectability from one disease to a similar disease, nor from one individual to another. This would allow treatment unique to that person's disease who does not share a class with other patients. But artificial intelligence and machine learning depend upon large data sets, upon population-level data.

Precision medicine treatment is not feasible because medical evidence is based on general tenets, induction, derived from current diagnostic criteria (perpetually undergoing refinements, clinical trials and epidemiology applicable to all or most patients within the disease class.)

Diseases are not stable entities but evolve during treatment to resist treatments, the modal nature is altered by treatment e.g. MRSA, influenza, cancers. the origin of tuberculosis in the middle East is at least 9000 years old1113 Hershkovitz, Israel; Donoghue, Helen D.; Minnikin, David E.; Besra, Gurdyal S.; Lee, Oona Y-C.; Gernaey, Angela M.; Galili, Ehud; Eshed, Vered; Greenblatt, Charles L. (15 October 2008)."Detection and Molecular Characterization of 9000-Year-Old Mycobacterium tuberculosis from a Neolithic Settlement in the Eastern Mediterranean". PLoS ONE. 3 (10): e3426. and common resistant mutations are numerous, $\operatorname{Thr}(\mathrm{ACA}), \operatorname{Asn}(\mathrm{AAC})$, Ile(ATC), $\operatorname{Thr}(\mathrm{ACT})$, Gly(GGC) inhA promoter -15T and evade drug therapy.

Just as coronaviruses have become the current entity to manifest evolution and transmission across species.

Malaria has defied cure based on the point mutations and gene amplifications e.g.crt, dhps, dhfr, mdr1, and even artemisinin resistant malaria mediated by point mutations attributable to Kelch $13.22^{14}$ Menard D, Fidock DA. Accelerated evolution and spread of multi-drug resistant Plasmodium falciparum takes down the latest first-line antimalarial drugs in southeast Asia. The Lancet, Vol.19, issue 9, p 916-17, 2019 15Zhao Y, Liu Z, Soe MT. et al Genetic variation associated with drug resistance markers in asymptomatic Plasmodium falciparum infection in Myanmar.Genes,Vol.10 Issue 9, p 692, 201916 Darwin C. The origin of Species. 
(Variorum text) Chap 14. P 760.

EBV sequesters in B cells and silently reproduces only to remain viable. Herpes hides in neural ganglia for many years until opportunity arises.

Our taxonomy of disease is based on observations that, given signs and symptoms, diseases reflect the relationship between the disease, its cause and resistance to interventions, natural and therapeutic, and the patient's pathophysiology; each patient manifests symptoms and signs that mimic, but do not match exactly those in others with the same disease.

Disease classification is a homeostatic cluster of features that distinguish it from related diseases. "We carve nature at its joints." accordingly.

Leukemia requires a predicate, a sortal predicate if you will, thus ALL, AML, CLL, B-cell lymphomas are categories of the disease, yet antigenic markers distinguish among these diseases to allow targeted therapy, e.g. based on cytogenetics and therapeutically on molecular markers KIT, FLT3-ITD, NPM1, CEBPA, PD-L-1, CD-19 perhaps CD30.

As our nominative classification of these diseases is based on testing for the antigen expressions, and as therapy induces changes in the cellular expressions, so tumor cells become resistant to further elimination by transmutations to acquire resistance; surviving clones in minimal residual disease (MRD.) Discrete classes, carved at their joints, provide more specific treatments. Or so it is hoped. The mechanistic approach of precision medicine is here applicable. But the measurements required to perform to characterize a condition are on a asymptotic curve.

Evolutionary changes in the body, dependent on both external and internal environments, allow diseases to escape the immune system as illustrated above. Tumor cells evolve to become refractory to chemotherapy. Loss of responsiveness to treatment with monoclonal antibodies (mAbs) such as

rituximab is a serious complication during therapy of B-cell malignancies but the mechanisms responsible for it are not well understood. Even CAR-T therapy directed against CD-19 has not shown success in all cases of leukemia and lymphoma; CD30 is a promising alternative antigen target. BTK inhibitors such as ibrutinid may play a similar role in some

non-Hodgkin's lymphomas.

Clones of tumor stem cells evolve to resist intervention.33

Molecular evolution, even in laboratory-controlled constant supporting conditions are determined by stochastic processes. Escherichia coli mutation and was measured over 60,000 generations showing only a slight decline in fitness; and these changes were balanced against multiple beneficial variants.4417.Good B.H., MaDonadl M.J. et al. The dynamics of molecular evolution over 60,000 generations. Nature 551, 2 November $2017, ; 4550$

Thus, generalizability (induction) is forfeited to particularity, sensitivity to specificity, and randomized controlled trials would suffer from lack of participation since each disease would be treated somewhat differently, depending on the definition based on the character of the disease, genetics, as well as on the sufferer. Thus, precision medicine would usurp the medical treatment available based on randomized controlled trials and would evolve into 'customized' treatment. Treatment protocols would be suited to the genomic markers of the disease and these degrees of difference might require different therapies. This would eventually and controversially obviate the need for randomized trials

\section{Research options :}

In place of RCTs precision clinical trials might include $\mathrm{n}$ of 1 , or even master protocols such as umbrella and basket trials. One SNP does not make an entirely new disease. That is the foundation of umbrella protocols, Tumor cells regulate antigenic self-expression in order to survive the treatment by protocol drugs, 
and they usually succeed. Even ALL is not vanquished, thus CAR-T Therapy. Yet which tumor cells to search? which fluid biopsies of CTCs or ct-DNA to sort and harvest and insert a chimeric antigen?

Disease persists or recurs:

Why do disease treatments fail, in the treatment of AML et al if the residual tumor cells are antigenically similar progeny of the originals? Simply the effect of mutagenic chemotherapy? Or subclones of the blast or progenitor population? Or do the blasts evolve and develop subsequent resistance to any and all therapeutic agents? AML is the best suited model for laboratory investigation as the disease mimics the accepted cancer stem cell model.

Controversially the definition of cancer stem cells (CSC) is not yet settled: is the CSC an initiating cell, a propagating cell, or a stem-like cell?

Evolutionary changes in the body, dependent on both the external and internal environment (chemo and immune-therapy), allow diseases to escape natural controls i.e. the immune system, and provide shields to further treatment. Sequencing of paired initial and relapse AML cells reveal relapse is reflected in minor genetic subclones initially present which survive chemotherapy. What are these cells?

Similarly, in lymphoma, tumor cells evolve to become refractory to chemotherapy, loss of responsiveness to treatment with monoclonal antibodies (mAbs) such as rituximab and that is a serious complication during therapy of B-cell malignancies, but the mechanisms responsible for it are not well understood.11

This is what one would expect from natural kinds such as ourselves; we evolve to survive ambient cultures e.g. obesity and Type II diabetes may be considered evolutionary alterations 22 a shift from simpler diets and activities.

The most important advance provided for in the concept of precision medicine initiative will be a more specific way to define and thus to understand disease, but it will not finally be therapeutically effective since natural kinds will not easily succumb to alteration of the interior milieu i. e. the treatment that will try to change this interior state.3312 Natural kinds will adapt to interventions as quickly as they can.

As precision medicine is aimed at understanding disease, we must be able to take advantage of survival distributions; if every disease is unique, there is no purchase for induction, nor path to generalize treatment If each case of lung cancer will require different immunomodulating drugs, 1344 and the cost for one year exceeds $\$ 150,000$, or CART therapy with axicabtagene costing $\$ 373,000$ for a single injection, how shall equitable distribution be established in the non-insured population?55 667714

"There is a grandeur in this view of life (and disease) with its several powers, having been originally breathed into a few forms or into one; and that whilst the planet has gone cycling on according to the fixed law of gravity, from so simple a beginning endless forms most beautiful and most wonderful have been, and are being, evolved." 8815

Declarations:

- Ethics approval and consent to participate not applicable

- Consent for publication: Not applicable

- Availability of data and material: not applicable

- Competing interests: none

- Funding: none

- Authors' contributions: single author

- Acknowledgements: Maine Medical Center Library

1. Torkamani a., Andersen KG, Steinhubi AR. High definition Medicine. Cell 2017,170 (5) p 828-843

2. Nagen M., Chen Y., Lovejoy CA., Artificial intelligence versus clinicians : systematic review of design, reporting standards, and claims of deep learning studies. BMJ 25 March 2020 p 368-381 
3 Quine WVO. "Natural Kinds" from Ontological Relativity and Other Essays.1969, pp.134-138

4 Manrai AK. Ioannidis JPA Kohan eIS Clinical Genomics from Pathogenicity Claims to Quantitative Risk Estimates JAMA March 22/29, 2016 Vol 315.No. 12

5. Laplane, Lucie "Cancer Stem Cells: philosophy and therapies" HUP 2016

6.Tannock IF, Hickman Ja "Limits to Personalized Cancer Medicine, NEJM.375:13 1259-1294.

7. Dilley RL, Verma, P, Cho NM et al: Break-induced telomere synthesis underlies alternative telomere maintenance. Nature $2016 ; 53.9$ 54-58

8. Hoffman EP Skipping toward Personalized Molecular Medicine NEJM 357; 26 December 27, 2007.

9. MacConaill LE. Brave-ish New World-What's needed to make precision oncology a practical reality, JAMA Oncology July 16, 2015

10. Joyner MJ Seven Quesitons for Personalized Medicine. JAMA sept 8, 2015 999-1000

11. Jamal-Hanjani M et al tracking the Evolution of Non-Small Cell Lung cancer NEJM 376;22 June 1, 2017

12. Shlush L. Tracing the origins of relapse in acute myeloid

leukaemia to stem cells Ieukemia Nature 547, 104-108 July 6, 2017

12. Hoffman E.P. Skipping toward Personalized Molecular Medicine. NEJM 357:26, Dec. 2020 